\title{
THE ROBIN PROBLEM FOR THE HÉNON EQUATION
}

\author{
HAIYANG HE
}

(Received 18 April 2012; accepted 3 April 2013; first published online 27 June 2013)

\section{Abstract}

In this paper, we consider the following Robin problem:

$$
\begin{cases}-\Delta u=|x|^{\alpha} u^{p}, & x \in \Omega, \\ u>0, & x \in \Omega, \\ \frac{\partial u}{\partial v}+\beta u=0, & x \in \partial \Omega\end{cases}
$$

where $\Omega$ is the unit ball in $\mathbb{R}^{N}$ centred at the origin, with $N \geq 3, p>1, \alpha>0, \beta>0$, and $v$ is the unit outward vector normal to $\partial \Omega$. We prove that the above problem has no solution when $\beta$ is small enough. We also obtain existence results and we analyse the symmetry breaking of the ground state solutions.

2010 Mathematics subject classification: primary 35J20; secondary $35 \mathrm{~J} 60$.

Keywords and phrases: Hénon equation, symmetry breaking, Robin problem, ground state solutions.

\section{Introduction}

Let us consider the following problem:

$$
\begin{cases}-\Delta u=|x|^{\alpha} u^{p}, & x \in \Omega, \\ u>0, & x \in \Omega, \\ \frac{\partial u}{\partial v}+\beta u=0, & x \in \partial \Omega\end{cases}
$$

where $\Omega$ is the unit ball in $\mathbb{R}^{N}$ centred at the origin, with $N \geq 3, p>1, \alpha>0, \beta>0$, and $v$ is the unit outward vector normal to $\partial \Omega$.

Problem (1.1) has different names depending on the different values of the parameter $\beta$. It is called a Dirichlet problem if $\beta=+\infty$, a Neumann problem if $\beta=0$ and a Robin problem if $0<\beta<+\infty$.

This work was supported by the National Natural Sciences Foundation of China (No. 11201140), the Key Project of Chinese Ministry of Education (No. 212120) and the Scientific Research Fund of Hunan Provincial Education Department.

(c) 2013 Australian Mathematical Publishing Association Inc. 0004-9727/2013 \$16.00 
When $\beta=0$, it is trivial since by integration by parts we can easily prove that (1.1) has no solution.

When $\beta=+\infty,(1.1)$ is reduced to the following problem:

$$
-\Delta u=|x|^{\alpha} u^{p}, \quad x \in \Omega, u=0, x \in \partial \Omega .
$$

Equation (1.2) was proposed by Hénon in [12] when he studied rotating stellar structures and is called the Hénon equation. A standard compactness argument shows that the infimum

$$
\inf _{u \in H_{0}^{1}(\Omega) \backslash\{0\}} \frac{\int_{\Omega}|\nabla u|^{2} d x}{\left(\int_{\Omega}|x|^{\alpha}|u|^{p+1} d x\right)^{2 /(p+1)}}
$$

is achieved for any $1<p<2 N /(N-2)-1$ and $\alpha>0$. In 1982, Ni proved in [14] that the infimum

$$
\inf _{u \in H_{0, \text { rad }}^{1}(\Omega) \backslash\{0\}} \frac{\int_{\Omega}|\nabla u|^{2} d x}{\left(\int_{\Omega}|x|^{\alpha}|u|^{p+1} d x\right)^{2 /(p+1)}}
$$

is achieved for any $p \in(1,(N+2+2 \alpha) /(N-2))$ by a function in $H_{0 \text {,rad }}^{1}(\Omega)$, the space of radial $H_{0}^{1}(\Omega)$ functions. Thus, radial solutions of (1.2) exist also for (Sobolev) supercritical exponents $p$.

A natural question is whether any minimiser of (1.3) must be radially symmetric in the range $1<p<(N+2) /(N-2)$ and $\alpha>0$. For $\alpha>0$, since the function $r \mapsto r^{\alpha}$ is increasing, neither rearrangement arguments nor the moving plane techniques of [9] can be applied. Therefore nonradial solutions could be expected. Numerical solutions obtained by Chen et al. [7] show that for fixed $p \in(1,(N+2) /(N-2))$ the ground state solution of problem (1.2) is nonradial if $\alpha$ is large enough. Smets et al. also proved in [17] some symmetry-breaking results for (1.2). They proved that minimisers of (1.3) (the so-called ground state solutions, or least energy solutions) cannot be radial for $\alpha$ large enough. As a consequence, (1.2) has at least two solutions when $\alpha$ is large. (See also [18].)

Further results on problem (1.2) can be found in [4-6, 18] for residual symmetry properties and asymptotic behaviour of ground states (for $p \rightarrow(N+2) /(N-2)$, or $\alpha \rightarrow \infty)$ and in $[2,15,16]$ for existence and multiplicity of nonradial solutions for critical, supercritical and slightly subcritical growth.

In [8], Gazzini et al. studied the Hénon equation with Neumann boundary conditions

$$
\begin{cases}-\Delta u+u=|x|^{\alpha} u^{p}, & x \in \Omega, \\ \frac{\partial u}{\partial v}=0, & x \in \partial \Omega, \\ u>0, & x \in \Omega .\end{cases}
$$

They proved that for any $p \in(1,(N+2+2 \alpha) /(N-2)),(1.4)$ also admits at least one radial solution. They pointed out for any $p \in(N /(N-2),(N+2) /(N-2))$, no ground state solution of (1.4) is radial provided $\alpha$ is large enough. 
Compared with problems (1.2) and (1.4), there are few results on the Robin problem for the Hénon equation. The purpose of this paper is to fill this gap and to point out a series of new, interesting and unexpected phenomena that arise in passing from problem (1.2) to problem (1.4).

To describe our results, we will investigate the functional $Q_{\alpha}(u): H^{1}(\Omega) \rightarrow \mathbb{R}$ defined by

$$
Q_{\alpha}(u)=\frac{\int_{\Omega}|\nabla u|^{2} d x+\beta \int_{\partial \Omega} u^{2} d \sigma}{\left(\int_{\Omega}|x|^{\alpha}|u|^{p+1} d x\right)^{2 /(p+1)}} .
$$

We will describe as 'ground states' the functions that minimise $Q_{\alpha}$ over $H^{1}(\Omega)$, while we reserve the term 'radial minimiser' for functions that minimise $Q_{\alpha}$ over $H_{\text {rad }}^{1}(\Omega)$.

The first result of this paper can be stated as the following theorem.

THeorem 1.1. If $1<p<(N+2) /(N-2)$ then there exists a positive number $\beta^{*}$ such that (1.1) has no solution for any $\beta \in\left(0, \beta^{*}\right)$.

The second result concerns the existence of a solution to (1.1). It may be presented as follows.

Theorem 1.2. If $\beta>\beta^{*}$, for any $\alpha>0$ and $p \in(1,(N+2+2 \alpha) /(N-2))$, there exists $u \in H_{\mathrm{rad}}^{1}(\Omega)$ such that

$$
Q_{\alpha}(u)=\inf _{v \in H_{\mathrm{rad}}^{1}(\Omega) \backslash\{0\}} Q_{\alpha}(v) .
$$

A suitable multiple of $u$ is a classical solution of (1.1).

The purpose of this paper is to study the symmetry properties of the ground states as $\alpha \rightarrow \infty$. The final result is as follows.

Theorem 1.3. If $\beta>\beta^{*}$, for any $p \in(N /(N-2),(N+2) /(N-2))$, no ground state of $Q_{\alpha}$ is radial provided $\alpha$ is large enough.

REMARK 1.4. Theorems 1.2 and 1.3 give a multiplicity result: for every $p \in(N /(N-2)$, $(N+2) /(N-2))$ and $\alpha$ large enough, (1.1) admits at least two solutions. One is radial and the other is the (nonradial) ground state.

This paper is organised as follows. In Section 2 we study the nonexistence result of (1.1). In Section 3 we establish the main existence result and carry out the asymptotic analysis of radial minimisers.

\section{The proof of Theorem 1.1}

Before giving our proof, we need the following lemmas.

LEMMa 2.1. If $u$ is a nonnegative solution of the equation

$$
-\Delta u=u^{p}, \quad x \in \mathbb{R}^{N},
$$

with $1<p<(N+2) /(N-2)$, then $u \equiv 0$. 
LEMMA 2.2. Let $u(x)$ be a nonnegative solution of

$$
-\Delta u=|x|^{\alpha} u^{p}, \quad x \in \mathbb{R}^{N},
$$

with $N \geq 3, \quad \alpha>-2$ and $p$ satisfying $1<p<(N+2) /(N-2), \quad p \neq(N+2+2 \alpha) /$ $(N-2)$. Then $u \equiv 0$.

Lemmas 2.1 and 2.2 are proved in [10].

Lemma 2.3. If $\alpha>0,1<p<(N+2) /(N-2)$, for $\beta$ small enough, then there exists a number $M>0$ independent of $\beta$ such that any solution $u=u_{\beta}$ of (1.1) satisfies

$$
\|u\|_{L^{\infty}(\bar{\Omega})} \leq M .
$$

Proof. Suppose that the conclusion is not true. Then there exist a sequence $\beta_{j} \rightarrow 0$ as $j \rightarrow \infty$, a corresponding sequence of solutions $u_{j}=u_{\beta_{j}}$ of (1.1) with $\beta=\beta_{j}$ and a sequence of points $x_{j} \in \bar{\Omega}$ such that

$$
M_{j}=\left\|u_{j}\right\|_{L^{\infty}(\bar{\Omega})}=u_{j}\left(x_{j}\right) \rightarrow \infty, \quad \text { as } j \rightarrow \infty .
$$

Suppose that for a subsequence of $j$ as $j \rightarrow \infty, x_{j} \rightarrow x_{0} \in \bar{\Omega}$. Here our reduction procedure breaks down into three cases: (1) $x_{0} \in \Omega, x_{0} \neq 0$; (2) $x_{0} \in \partial \Omega$; (3) $x_{0}=0$. Cases (1) and (2) will be treated simultaneously; case (3) is slightly harder and is treated separately.

Cases (1) and (2). Let $\lambda_{j}$ be a sequence of positive numbers defined by $\lambda_{j}^{2 /(p-1)} M_{j}=$ 1 and $y=x-x_{j} / \lambda_{j}$. Define scaled functions

$$
v_{j}(y)=\lambda_{j}^{2 /(p-1)} u_{j}(x)
$$

and the domain $\Omega_{j}=\left\{y \in \mathbb{R}^{N} \mid \lambda_{j} y+x_{j} \in \Omega\right\}$. Since $M_{j} \rightarrow+\infty$, we have $\lambda_{j} \rightarrow 0$ as $j \rightarrow+\infty$. It is easy to see that $v_{j}(y)$ satisfies

$$
\begin{cases}-\Delta v_{j}=\left|\lambda_{j} y+x_{j}\right|^{\alpha} v_{j}^{p}, & y \in \Omega_{j}, \\ \frac{\partial v_{j}}{\partial v}+\beta_{j} \lambda_{j} v_{j}=0, & y \in \partial \Omega_{j}, \\ 0<v_{j} \leq 1, \quad v_{j}(0)=1, & y \in \Omega_{j} .\end{cases}
$$

Let $2 d$ denote the distance of $x_{0}$ to $\partial \Omega$. For large $j, v_{j}(y)$ is well defined in the ball $B_{d / \lambda_{j}}(0)$, and

$$
\sup _{y \in B_{d / \lambda_{j}}(0)} v_{j}(y)=v_{j}(0)=1 .
$$

Since $0<v_{j}(y) \leq 1$, given any radius $R$ such that $B_{R}(0) \subset B_{d / \lambda_{j}}(0)$, by the $L^{p}$ estimates in the theory of elliptic equations (see [13]), we can find uniform bounds for $\left\|v_{j}\right\|_{w^{2, p}\left(B_{R}(0)\right)}, p>N$. Choosing $j$ large enough, we obtain by Morrey's theorem [13] that $\left\|v_{j}\right\|_{C^{2, \gamma}\left(B_{R}(0)\right)}, 0<\gamma<1$, is also uniformly bounded. Hence we may have

$$
v_{j} \rightarrow v \quad \text { in } w^{2, p} \cap C^{1, \gamma}(p>N, 0<\gamma<1) \quad \text { on } B_{R}(0)
$$


for $j$ large enough. Furthermore, since $y \in B_{R}(0)$,

$$
\lambda_{j} y+x_{j} \rightarrow x_{0} \quad \text { as } j \rightarrow \infty .
$$

As in [13], we may assume that $\left\{v_{j}\right\}$ converges uniformly on any compact domain of $D\left(\mathbb{R}^{N}\right.$ or $\left.\mathbb{R}_{+}^{N}\right)$ to a function $v$. Then $v(y)$ is a solution of

$$
\begin{cases}-\Delta v=\left|x_{0}\right|^{\alpha} v^{p}, & y \in D, \\ \frac{\partial v}{\partial v}=0, & y \in \partial D\left(D=\mathbb{R}_{+}^{N}\right), \\ 0<v \leq 1, v(0)=1, & y \in D .\end{cases}
$$

If $D=\mathbb{R}^{N}$, then $\tilde{v}=\left|x_{0}\right|^{\alpha /(p-1)} v$ satisfies

$$
\begin{cases}-\Delta \tilde{v}=\tilde{v}^{p}, & y \in \mathbb{R}^{N}, \\ \tilde{v}>0, & y \in \mathbb{R}^{N}, \\ \tilde{v}(0)=\left|x_{0}\right|^{\alpha /(p-1)} . & \end{cases}
$$

This contradicts Lemma 2.1.

If $D=\mathbb{R}_{+}^{N}$, then the function $\tilde{v}$ defined by

$$
\tilde{v}\left(y_{1}, y_{2}, \ldots, y_{n}\right)= \begin{cases}v\left(y_{1}, y_{2}, \ldots, y_{N-1}, y_{N}\right) & \text { if } y \in \mathbb{R}_{+}^{N}, \\ v\left(y_{1}, y_{2}, \ldots, y_{N-1},-y_{N}\right) & \text { if } y \in \mathbb{R}_{-}^{N},\end{cases}
$$

satisfies

$$
\begin{cases}-\Delta \tilde{v}=\tilde{v}^{p}, & y \in \mathbb{R}^{N}, \\ \tilde{v}>0, & y \in \mathbb{R}^{N}, \\ \tilde{v}(0)=1 . & \end{cases}
$$

This also contradicts Lemma 2.1.

Case (3). $x_{0}=0$. In this case we choose $\lambda_{j}$ such that $\lambda_{j}^{(2+\alpha) /(p-1)} M_{j}=1$ and define the scaled functions

$$
v_{j}(y)=\lambda_{j}^{(2+\alpha) /(p-1)} u_{j}(x), \quad y=\frac{x-x_{j}}{\lambda_{j}} .
$$

We also have $\lambda_{j} \rightarrow 0$ as $j \rightarrow \infty$, and $v_{j}(y)$ satisfies

$$
\begin{cases}-\Delta v_{j}=\left|y+\frac{x_{j}}{\lambda_{j}}\right|^{\alpha} v_{j}^{p}, & y \in \Omega_{j}, \\ \frac{\partial v_{j}}{\partial v}+\beta_{j} \lambda_{j} v_{j}=0, & y \in \partial \Omega_{j}, \\ 0<v_{j} \leq 1, v_{j}(0)=1, & y \in \Omega_{j},\end{cases}
$$

where $\Omega_{j}=\lambda_{j}^{-1}\left(\Omega-x_{j}\right)$. 
For large $j, v_{j}(y)$ is also well defined in $B_{d / \lambda_{j}}(0)(2 d=\operatorname{dist}(0, \partial \Omega))$. The difficulty lies in the fact that $\left|x_{j}\right| / \lambda_{j}$ might be unbounded and $\left|x_{j}\right| / \lambda_{j} \rightarrow+\infty$ along a subsequence $j \rightarrow \infty$. From [11], we will have

$$
v_{j}(y) \leq \frac{C}{\mid y+\frac{x_{j}}{\lambda_{j}}(2+\alpha) /(p-1)} \quad \text { near } y=0,
$$

where $C$ is a uniform constant. Since $v_{j}(0)=1$,

$$
\frac{\left|x_{j}\right|}{\lambda_{j}} \leq C_{1}
$$

Thus, up to a subsequence, we have $x_{j} / \lambda_{j} \rightarrow P_{0}$ with $\left|P_{0}\right| \leq C_{1}<+\infty$. As in cases (1) and (2),

$$
v_{j} \rightarrow v \quad \text { in } w^{2, p} \cap C^{1, \gamma}(p>N, 0<\gamma<1)
$$

on any compact subset in $\mathbb{R}^{N}$ and $v$ satisfies

$$
\begin{cases}-\Delta v=\left|y+P_{0}\right|^{\alpha} v^{p}, & y \in \mathbb{R}^{N}, \\ 0<v \leq 1, v(0)=1, & y \in \mathbb{R}^{N} .\end{cases}
$$

By a translation $y+P_{0} \mapsto y$ and

$$
\left\{\begin{array}{l}
-\Delta v=|y|^{\alpha} v^{p}, \quad y \in \mathbb{R}^{N}, \\
v\left(P_{0}\right)=1 .
\end{array}\right.
$$

By Lemma 2.2, we conclude that $v \equiv 0$, which contradicts $v\left(P_{0}\right)=1$. This completes the proof of Lemma 2.3.

Proof of Theorem 1.1. We argue by contradiction. Suppose that the conclusion of Theorem 1.1 is false. Then there exists a sequence $\beta_{j} \rightarrow 0^{+}$as $j \rightarrow+\infty$ such that (1.1) with $\beta=\beta_{j}$ has at least one positive solution $u_{\beta_{j}}$. By Lemma 2.3 and the standard elliptic estimate, there exists a positive constant $C$ independent of $j$ such that $\left\|u_{\beta_{j}}\right\|_{C^{2, \gamma}(\bar{\Omega})} \leq C$. Hence, up to a subsequence, we may assume that

$$
u_{\beta_{j}} \rightarrow u \text { in } C^{2}(\Omega)
$$

as $j \rightarrow \infty$ and $u$ is a solution of the following problem:

$$
\begin{cases}-\Delta u=|x|^{\alpha} u^{p}, & x \in \Omega, \\ \frac{\partial u}{\partial v}=0, & x \in \partial \Omega, \\ u \geq 0, & x \in \Omega .\end{cases}
$$

However, it is easy to see that (2.1) has no solutions. This is a contradiction. 


\section{Radial minimisers and their asymptotic analysis}

Let $\Omega$ be the unit ball in $\mathbb{R}^{N}$ with $N \geq 3$; the numbers $2^{*}=2 N /(N-2)$ and $2_{*}=$ $(2 N-2) /(N-2)$ are the critical exponents for the embedding of $H^{1}(\Omega)$ into $L^{p}(\Omega)$ and $L^{q}(\partial \Omega)$, respectively.

If we denote by $\lambda_{1}^{\beta}(\Omega)$ the first eigenvalue of

$$
\begin{cases}-\Delta \varphi=\lambda \varphi, & x \in \Omega \\ \frac{\partial \varphi}{\partial v}+\beta \varphi=0, & x \in \partial \Omega\end{cases}
$$

then

$$
\int_{\Omega}|\nabla v|^{2} d x+\beta \int_{\partial \Omega} v^{2} d \sigma \geq \lambda_{1}^{\beta}(\Omega) \int_{\Omega} v^{2} d x \quad \text { for any } v \in H^{1}(\Omega) .
$$

Accordingly,

$$
\begin{aligned}
\int_{\Omega}\left(|\nabla v|^{2}+v^{2}\right) d x & \leq \int_{\Omega}|\nabla v|^{2} d x+\frac{1}{\lambda_{1}^{\beta}(\Omega)} \int_{\Omega}|\nabla v|^{2} d x+\frac{\beta}{\lambda_{1}^{\beta}(\Omega)} \int_{\partial \Omega} v^{2} d \sigma \\
& \leq\left(1+\frac{1}{\lambda_{1}^{\beta}(\Omega)}\right)\left(\int_{\Omega}|\nabla v|^{2} d x+\beta \int_{\partial \Omega} v^{2} d \sigma\right) .
\end{aligned}
$$

By trace inequalities,

$$
\int_{\partial \Omega} v^{2} d \sigma \leq C \int_{\Omega}\left(|\nabla v|^{2}+v^{2}\right) d x
$$

which implies that

$$
\int_{\Omega}|\nabla v|^{2} d x+\beta \int_{\partial \Omega} v^{2} d \sigma \leq C \int_{\Omega}\left(|\nabla v|^{2}+v^{2}\right) d x \quad \text { for any } v \in H^{1}(\Omega) .
$$

Hence, the norm $\|u\|_{H^{1}(\Omega)}=\left(\int_{\Omega}|\nabla u|^{2}+u^{2} d x\right)^{1 / 2}$ is equivalent to

$$
\left(\int_{\Omega}|\nabla u|^{2} d x+\beta \int_{\partial \Omega} u^{2} d \sigma\right)^{1 / 2}:=\|u\|
$$

Now we study the function $Q_{\alpha}(u): H^{1}(\Omega) \rightarrow \mathbb{R}$ defined by

$$
Q_{\alpha}(u)=\frac{\int_{\Omega}|\nabla u|^{2} d x+\beta \int_{\partial \Omega} u^{2} d \sigma}{\left(\int_{\Omega}|x|^{\alpha}|u|^{p+1} d x\right)^{2 /(p+1)}}=\frac{\|u\|}{\left(\int_{\Omega}|x|^{\alpha}|u|^{p+1} d x\right)^{2 /(p+1)}} .
$$

The functional is well defined and of class $C^{2}$ over $H^{1}(\Omega)$ if $p \leq(N+2) /(N-2)$. We shall see in a while that its restriction to $H_{\text {rad }}^{1}(\Omega)$, which is the space of radial functions in $H^{1}(\Omega)$, is still well defined and $C^{2}$ for a much wider interval of $p$.

In order to establish the main existence results, we need the following lemmas. 
Lemma 3.1. There exists a positive constant $C$ such that, for all $u \in H_{\mathrm{rad}}^{1}(\Omega)$,

$$
|u(x)| \leq C \frac{\|u\|}{|x|^{(N-2) / 2}}, \quad x \in \Omega \backslash\{0\} .
$$

Proof. This follows by the radial lemma of [14].

From Lemma 3.1 and similar to the method for the Dirichlet problem obtained in [14], we have the following result.

Lemma 3.2. The space $H_{\mathrm{rad}}^{1}(\Omega)$ embeds compactly into $L^{p}\left(\Omega,|x|^{\alpha} d x\right)$ for any $p \in$ $\left[1,2^{*}+2 \alpha /(N-2)\right)$, where $L^{p}\left(\Omega,|x|^{\alpha} d x\right)$ is the space of $L^{p}$ functions on $\Omega$ with respect to the measure $|x|^{\alpha} d x$.

We are now ready to give the main existence result.

Theorem 3.3. If $\beta>\beta^{*}$, for any $\alpha>0, p \in(1,(N+2+2 \alpha) /(N-2))$, there exists $u \in H_{\mathrm{rad}}^{1}(\Omega)$ such that

$$
Q_{\alpha}(u)=\inf _{v \in H_{\mathrm{rad}}^{1}(\Omega)} Q_{\alpha}(v) .
$$

Proof. This follows by Lemma 3.2 and similarly to the proof of [14].

Corollary 3.4. For any $\alpha, \beta$ and $p$ as in Theorem 3.3, (a suitable multiple of) the minimiser $u$ is a classical solution of the problem

$$
\begin{cases}-\Delta u=|x|^{\alpha} u^{p}, & x \in \Omega, \\ \frac{\partial u}{\partial v}+\beta u=0, & x \in \partial \Omega .\end{cases}
$$

Moreover, $u$ is strictly positive in $\bar{\Omega}$.

Proof. From the symmetric criticality principle and standard elliptic regularity, we know that $u$ is a classical solution of (3.1). Replacing $u$ by $|u|$, we may also assume that $u$ is nonnegative. By the maximum principle, we have $u>0$ in $\Omega$. Assume that there exists $x_{0} \in \partial \Omega$ such that $u\left(x_{0}\right)=0$. Then $(\partial u / \partial v)\left(x_{0}\right)<0$ by the Hopf lemma, but the boundary condition implies that $(\partial u / \partial v)\left(x_{0}\right)=0$, a contradiction. The result follows.

For $\beta>\beta^{*}, \alpha>0$ and $p \in(1,(N+2+2 \alpha) /(N-2))$, let

$$
S_{\alpha}^{\mathrm{rad}}=\min _{u \in H_{\mathrm{rad}}^{1}(\Omega) \backslash\{0\}} Q_{\alpha}(u) .
$$

Then any (positive) minimiser $u_{\alpha}$ of $Q_{\alpha}$ over $H_{\text {rad }}^{1}(\Omega)$, when normalised by $\left\|u_{\alpha}\right\|=1$, satisfies

$$
\begin{cases}-\Delta u_{\alpha}=\left(S_{\alpha}^{\mathrm{rad}}\right)^{(p+1) / 2}|x|^{\alpha} u_{\alpha}^{p}, & x \in \Omega, \\ \frac{\partial u_{\alpha}}{\partial v}+\beta u_{\alpha}=0, & x \in \partial \Omega .\end{cases}
$$

Now we will consider the asymptotic behaviour of $S_{\alpha}^{\mathrm{rad}}$ and $u_{\alpha}$ as $\alpha \rightarrow \infty$. We begin with a fundamental result. 
Lemma 3.5. If $p \in(1,(N+2) /(N-2))$, the asymptotic relation

$$
(\alpha+N) \int_{\Omega}|x|^{\alpha}|u|^{p+1} d x=\int_{\partial \Omega}|u|^{p+1} d \sigma+o(1), \quad \text { as } \alpha \rightarrow \infty,
$$

holds uniformly on bounded subsets of $H_{\mathrm{rad}}^{1}(\Omega)$.

Proof. This result can be found in [8].

From $[1,3]$, we have the following lemmas.

Lemma 3.6. The first eigenvalue $\lambda_{1}(\Omega)$ of the eigenvalue problem

$$
\begin{cases}-\Delta \varphi=0, & x \in \Omega \\ \frac{\partial \varphi}{\partial v}+\beta \varphi=\lambda \varphi, & x \in \partial \Omega\end{cases}
$$

is positive, and the first eigenfunction $\varphi_{1}$, corresponding to $\lambda_{1}(\Omega)$, does not vanish in $\bar{\Omega}$. Moreover, $\lambda_{1}(\Omega)=\delta_{1}(\Omega)+\beta$, where $\delta_{1}(\Omega)$ is the Steklov eigenvalue for $-\Delta$.

Lemma 3.7. The first eigenvalue $\lambda_{1}(\Omega)$ is simple. Let $\varphi_{1}, \varphi_{2}$ be two eigenfunctions associated with $\lambda_{1}(\Omega)$. Then there exists $C$ such that $\varphi_{1}=C \varphi_{2}$. Moreover, $\varphi_{1}$ is unique up to a constant factor and it is radial.

In the statement of the next result, $\lambda_{1}$ and $\varphi_{1}$, which is positive in $\bar{\Omega}$ and normalised by $\left\|\varphi_{1}\right\|=1$, are the first eigenvalue and eigenfunction of problem (3.2), respectively.

Theorem 3.8. Let $p \in(1,(N+2) /(N-2))$ and let $u_{\alpha}$, with $\left\|u_{\alpha}\right\|=1$, be the minimiser of $Q_{\alpha}$ over $H_{\mathrm{rad}}^{1}(\Omega)$ such that $S_{\alpha}^{\mathrm{rad}}=Q_{\alpha}\left(u_{\alpha}\right)$. Then, as $\alpha \rightarrow \infty$,

$$
S_{\alpha}^{\mathrm{rad}} \sim(\alpha+N)^{2 /(p+1)}|\partial \Omega|^{1-2 /(p+1)} \lambda_{1} .
$$

Proof. We adapt an argument in [8]. Let $u \in H_{\text {rad }}^{1}(\Omega)$ with $u \geq 0,\|u\|=1$. By Lemma 3.5, as $\alpha \rightarrow \infty$,

$$
\begin{aligned}
\frac{Q_{\alpha}(u)}{(\alpha+N)^{2 /(p+1)}} & =\frac{1}{\left((\alpha+N) \int_{\Omega}|x|^{\alpha}|u|^{p+1} d x\right)^{2 /(p+1)}} \\
& =\frac{1}{\left(\int_{\partial \Omega}|u|^{p+1} d \sigma+o(1)\right)^{2 /(p+1)}} \\
& =\frac{1}{\left(\int_{\partial \Omega}|u|^{p+1} d \sigma\right)^{2 /(p+1)}}+o(1),
\end{aligned}
$$

where $o(1)$ does not depend on $u$. Since $u$ is radial,

$$
\left(\int_{\partial \Omega}|u|^{p+1} d \sigma\right)^{2 /(p+1)}=|\partial \Omega|^{2 /(p+1)-1} \int_{\partial \Omega} u^{2} d \sigma .
$$


Now let $u=u_{\alpha}$, so

$$
\begin{aligned}
\frac{S_{\alpha}^{\mathrm{rad}}}{(\alpha+N)^{2 /(p+1)}}=\frac{Q_{\alpha}\left(u_{\alpha}\right)}{(\alpha+N)^{2 /(p+1)}} & =\frac{1}{\left(\int_{\partial \Omega}\left|u_{\alpha}\right|^{p+1} d \sigma\right)^{2 /(p+1)}}+o(1) \\
& \geq|\partial \Omega|^{1-2 /(p+1)} \min _{v \in H_{\mathrm{rad}}^{1}(\Omega),\|v\|=1} \frac{1}{\int_{\partial \Omega} v^{2} d \sigma}+o(1) \\
& =|\partial \Omega|^{1-2 /(p+1)} \lambda_{1}+o(1) .
\end{aligned}
$$

On the other hand, for any $u \in H_{\text {rad }}^{1}(\Omega)$ with $\|u\|=1$,

$$
\begin{aligned}
\frac{S_{\alpha}^{\mathrm{rad}}}{(\alpha+N)^{2 /(p+1)}} & =\frac{Q_{\alpha}\left(u_{\alpha}\right)}{(\alpha+N)^{2 /(p+1)}} \leq \frac{Q_{\alpha}(u)}{(\alpha+N)^{2 /(p+1)}} \\
& \leq|\partial \Omega|^{1-2 /(p+1)} \frac{1}{\int_{\partial \Omega} u^{2} d \sigma}+o(1) \\
& =|\partial \Omega|^{1-2 /(p+1)} \lambda_{1}+o(1)
\end{aligned}
$$

and, choosing $u=\varphi_{1}$,

$$
\frac{S_{\alpha}^{\mathrm{rad}}}{(\alpha+N)^{2 /(p+1)}} \leq|\partial \Omega|^{1-2 /(p+1)} \lambda_{1}+o(1) .
$$

Thus (3.3) is proved.

Theorem 3.9. If $\beta>\beta^{*}$ and $p \in(N /(N-2),(N+2) /(N-2))$, then, for $\alpha$ large enough (depending on $p$ and $\beta$ ),

$$
\min _{u \in H^{1}(\Omega) \backslash\{0\}} \frac{\|u\|}{\left(\int_{\Omega}|x|^{\alpha}|u|^{p+1} d x\right)^{2 /(p+1)}}<\min _{u \in H_{\mathrm{rad}}^{1}(\Omega) \backslash\{0\}} \frac{\|u\|}{\left(\int_{\Omega}|x|^{\alpha}|u|^{p+1} d x\right)^{2 /(p+1)}} .
$$

Proof. Choose a nonnegative function $u \in C_{0}^{1}(\Omega)$ and extend it to zero outside $\Omega$. Define $v_{\alpha}(x)=u\left(\alpha\left(x-x_{\alpha}\right)\right)$, where $x_{\alpha}=(1-1 / \alpha, 0, \ldots, 0)$. Then

$$
\int_{\Omega}|x|^{\alpha}\left|u_{\alpha}\right|^{p+1} d x \geq\left(1-\frac{2}{\alpha}\right)^{\alpha} \alpha^{-N} \int_{\Omega}|u|^{p+1} d x
$$

and

$$
Q_{\alpha}\left(u_{\alpha}\right) \leq \frac{\alpha^{2-N} \int_{\Omega}|\nabla u|^{2} d x}{\alpha^{-2 N /(p+1)}\left(1-\frac{2}{\alpha}\right)^{2 \alpha /(p+1)}\left(\int_{\Omega} u^{p+1} d x\right)^{2 /(p+1)}} \leq C \alpha^{2-N+2 N /(p+1)} .
$$

By Theorem 3.8, we know that $S_{\alpha}^{\mathrm{rad}} \sim \alpha^{2 /(p+1)}$. Since $2-N+2 N /(p+1)<2 /(p+1)$ for all $p \in(N /(N-2),(N+2) /(N-2))$, we see that (3.4) holds for $\alpha$ large enough. 


\title{
References
}

[1] G. Auchmuty, 'Steklov eigenproblems and the representation of solutions of elliptic boundary value problems', Numer. Funct. Anal. Optim. 25(3-4) (2004), 321-348.

[2] M. Badiale and E. Serra, 'Multiplicity results for the supercritical Hénon equation', Adv. Nonlinear Stud. 4 (2004), 453-467.

[3] C. Bandle, Isoperimetric Inequalities and Applications, Monographs and Studies in Mathematics, 7 (Pitman, Boston, 1980).

[4] J. Byeon and Z.-Q. Wang, 'On the Hénon equation: asymptotic profile of ground state I', Ann. Inst. H. Poincaré Anal. Non Linéaire 23 (2006), 803-828.

[5] J. Byeon and Z.-Q. Wang, 'On the Hénon equation: asymptotic profile of ground state II', J. Differential Equations 216 (2005), 78-108.

[6] D. Cao and S. Peng, 'The asymptotic behavior of the ground state solutions for Hénon equation', J. Math. Anal. Appl. 278 (2003), 1-17.

[7] G. Chen, W. M. Ni and J. Zhou, 'Algorithms and visualization for solutions of nonlinear elliptic equations', Internat. J. Bifur. Chaos Appl. Sci. Engrg. 10 (2000), 1565-1612.

[8] M. Gazzini and E. Serra, 'The Neumann problem for the Hénon equation, trace inequalities and Steklov eigenvalues', Ann. Inst. H. Poincaré Anal. Non Linéaire 25 (2008), 281-302.

[9] B. Gidas, W. M. Ni and L. Nirenberg, 'Symmetry of positive solutions of nonlinear elliptic equations in $\mathbb{R}^{N}$, in: Mathematical Analysis and Applications, Part A, Advances in Mathematics, Supplementary Studies, 7 (Academic Press, New York, 1981), 369-402.

[10] B. Gidas and J. Spruck, 'A priori bounds for positive solutions of nonlinear elliptic equations', Comm. Partial Differential Equations 8 (1981), 883-901.

[11] B. Gidas and J. Spruck, 'Global and local behavior of positive solutions of nonlinear elliptic equations', Comm. Pure Appl. Math. 34 (1981), 525-598.

[12] M. Hénon, 'Numerical experiments on the stability of spherical stellar systems', Astronom. Astrophys. Lib. 24 (1973), 229-238.

[13] C. B. Morrey, Multiple Integrals in the Calculus of Variations (Springer, New York, 1966).

[14] W. M. Ni, 'A nonlinear Dirichlet problem on the unit ball and its applications', Indiana Univ. Math. J. 31 (1982), 801-807.

[15] A. Pistoia and E. Serra, 'Multi-peak solutions for the Hénon equation with slightly subcritical growth', Math. Z. 256 (2007), 75-97.

[16] E. Serra, 'Nonradial positive solutions for the Hénon equation with critical growth', Calc. Var. Partial Differential Equations 23 (2005), 301-326.

[17] D. Smets, J. B. Su and M. Willem, 'Nonradial ground states for the Hénon equation', Commun. Contemp. Math. 4 (2002), 467-480.

[18] D. Smets and M. Willem, 'Partial symmetry and asymptotic behavior for some elliptic variational problems', Calc. Var. Partial Differential Equations 18 (2003), 57-75.

\author{
HAIYANG HE, College of Mathematics and Computer Science, \\ Key Laboratory of High Performance Computing and \\ Stochastic Information Processing (Ministry of Education of China), \\ Hunan Normal University, Changsha, Hunan 410081, PR China \\ e-mail: hehy917@hotmail.com
}

